\title{
ViP2P: Efficient XML Management in DHT Networks
}

\author{
Konstantinos Karanasos ${ }^{1}$, Asterios Katsifodimos ${ }^{1}$, \\ Ioana Manolescu ${ }^{1}$, and Spyros Zoupanos ${ }^{2}$ \\ 1 Inria Saclay-île de France and LRI, Université Paris Sud-11 \\ 2 Max-Planck Institut für Informatik, Saarbrücken, Germany \\ firstname.lastname@inria.fr
}

\begin{abstract}
We consider the problem of efficiently sharing large volumes of XML data based on distributed hash table overlay networks. Over the last three years, we have built ViP2P (standing for Views in Peerto-Peer), a platform for the distributed, parallel dissemination of XML data among peers. At the core of $\mathrm{ViP} 2 \mathrm{P}$ stand distributed materialized $X M L$ views, defined as XML queries, filled in with data published anywhere in the network, and exploited to efficiently answer queries issued by any network peer. ViP2P is one of the very few fully implemented $\mathrm{P} 2 \mathrm{P}$ platforms for XML sharing, deployed on hundreds of peers in a WAN. This paper describes the system architecture and modules, and the engineering lessons learned. We show experimental results, showing that our choices, outperf related systems by orders of magnitude in terms of data volumes, network size and data dissemination throughput.
\end{abstract}

Keywords: P2P, XML, DHT, distributed views.

\section{Introduction}

We consider the large-scale management of distributed XML data in a peer-topeer $(\mathrm{P} 2 \mathrm{P})$ setting. To provide users with precise and complete answers to their requests for information, we assume that the requests are formulated by means of a structured query language, and that the system must return complete results. That is, if somewhere in the distributed peer network, an answer to a given query exists, the system will find it and include it in the query result. Thus, we consider P2P XML data management based on a structured peer-to-peer network, more specifically, a distributed hash table (or DHT, in short).

In this setting, users may formulate two kinds of information requests. First, they may want to subscribe to interesting data anywhere in the network, that were published before or after the subscription is recorded in the system. We need to ensure that results are eventually returned as soon as possible, following the publication of a matching data source. Second, users may formulate ad-hoc (snapshot) queries, by which they just seek to obtain as fast as possible the results which have already been published in the network. 
The challenges raised by a DHT-based XML data management platform are: (i) building a distributed resource catalog, enabling data producers and consumers to "meet" in an information sharing space; such a catalog is needed both for subscription and ad-hoc queries; $(i i)$ efficiently distributing the data of the network to consumers that have subscribed to it, and; (iii) providing efficient distributed query evaluation algorithms for answering ad-hoc queries fast.

Over the last three years, we have invested more than 6 man-years building the ViP2P1 platform to address these challenges. Importantly, ViP2P uses subscriptions as views: results of long-running subscription queries are stored by the subscriber peers and re-used to answer subsequent ad-hoc queries.

A critical engineering issue when deploying XML data management applications on a DHT is the division of tasks between the DHT and the upper layers. In ViP2P, we chose to load the DHT layer as little as possible, and keep the heavyweight query processing operations in the data management layer and outside the DHT. This has enabled us to build and efficiently deploy an important-size system (70.000 lines of Java code), which we show scales on up to 250 computers in a WAN, and hundreds of GBs of XML data.

Several DHT-based XML data management platforms [3911] are only concerned with locating in the $\mathrm{P} 2 \mathrm{P}$ networks the documents relevant for a query. All the peers which may hold results then locally evaluate the query, leading to high query traffic and peer overload. In contrast, as in 1268, ViP2P answers queries over a global XML database distributed in a P2P network.

In this paper, we make the following contributions. $(i)$ We present a scalable, end-to-end architecture of one of the very few DHT-based XML sharing platforms actually implemented. From a system engineering perspective, we believe this is a useful addition to the corpus of existing DHT-based XML data management literature which has focused more on indexing and filtering algorithms, and less on system aspects. (ii) We present an experimental study of XML dissemination to DHT-based subscriptions, show which network parameters impact its performance, and demonstrate that ViP2P outperforms competitor systems by orders of magnitude in terms of published data volumes and throughput.

In the sequel, Section 2 presents an overview of the platform and Section 3 the architecture of a ViP2P peer. Section 4 experimentally demonstrate the platform scalability; many more experiments can be found in [4]. We then conclude.

\section{Platform Overview}

XML data flows in ViP2P can be summarized as follows. XML documents are published independently and autonomously by any peer. Peers can also formulate subscriptions, or long-running queries, potentially matching documents published before, or after the subscriptions. The results of each subscription query are stored at the peer defining the subscription, and the definition of it is indexed in the peer network. Finally, peers can ask ad-hoc queries, which are answered in a snapshot fashion (based on the data available in the network so far) by

${ }^{1}$ http://vip2p.saclay.inria.fr 

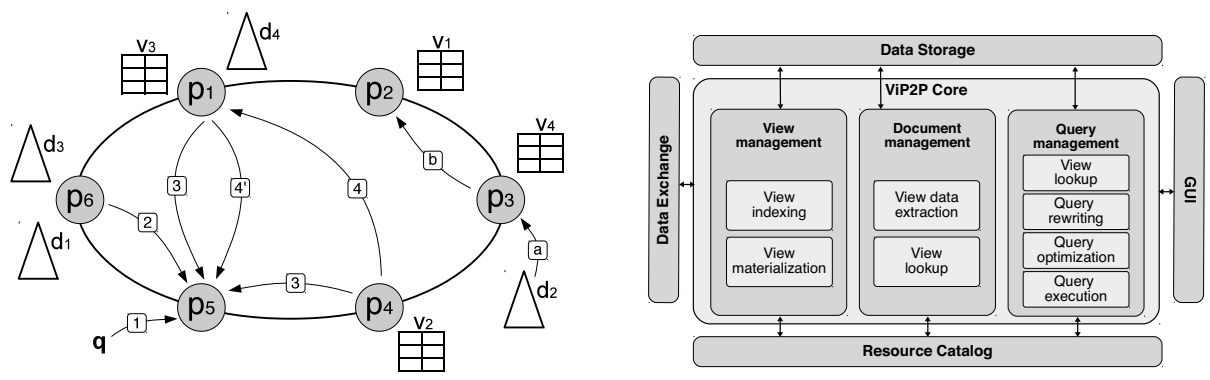

Fig. 1. System overview (left); Architecture of a ViP2P peer (right)

exploiting the existing subscriptions, which can be seen as materialized views. In this Section, we detail the overall process via an example.

A sample ViP2P instance over six peers is depicted in Figure 1 (left). In the Figure, XML documents are denoted by triangles, whereas views are denoted by tables, hinting to the fact that they contain sets of tuples.

For ease of explanation, we make the following naming conventions for the remainder of this paper: publisher is a peer which publishes an XML document, consumer is a peer which defines a subscription and stores its results (or, equivalently, the respective materialized view) and query peer is a peer which poses an ad-hoc query. Clearly, a peer can play any subset of these roles simultaneously.

View Publication. A ViP2P view is a long-running subscription query that any peer can freely define. In the sequel, we will refer to long-running subscription queries as materialized views or just views. The definition (i.e., the actual query) of each newly created view is indexed in the DHT network. For instance, assume peer $p_{2}$ in Figure 1 (left) publishes the view $v_{1}$, defined by the XPath query //bibliography//book[contains(.,'Databases $\left.\left.{ }^{\prime}\right)\right]$. The view requires all the books items from a bibliography containing the word 'Databases'. ViP2P indexes $v_{1}$ by inserting in the DHT the following three (key, value) pairs: (bibliography, $\left.v_{1} @ p_{2}\right)$, (book, $v_{1} @ p_{2}$ ) and ('Databases', $\left.v_{1} @ p_{2}\right)$. Here, $v_{1} @ p_{2}$ encapsulates the structured query defining $v_{1}$, and a pointer to the concrete database at peer $p_{2}$ where $v_{1}$ data is stored. As will be shown below, all existing and future documents that can affect $v_{1}$, push the corresponding data to its database.

Peers look up views in the DHT in two situations: when publishing documents, and when issuing ad-hoc queries. We detail this below.

Document Publication. When publishing a document, each peer is in charge of identifying the views within the whole network to which its document may contribute. For instance, in Figure1left (step a), peer $p_{3}$ publishes the document $d_{2}$. Peer $p_{3}$ extracts from $d_{2}$ all distinct element names and all keywords. For each such element name or keyword $k, p_{3}$ looks up in the DHT for view definitions associated to $k$. Assume that document $d_{2}$ contains data matching the view $v_{1}$ as it contains the element names bibliography and book, as well as the word 'Databases', thus $p_{3}$, learns about the existence of $v_{1}$ (step b). In the publication 
example above, $p_{3}$ extracts from $d_{2}$ the results matching $v_{1}$; from now on, we will use the notation $v_{1}\left(d_{2}\right)$ to designate such results. Peer $p_{3}$ sends $v_{1}\left(d_{2}\right)$ to $p_{2}$ (step c), which adds them to the database storing $v_{1}$ data.

Ad-Hoc Query Answering. ViP2P peers may pose ad-hoc queries, which must be evaluated immediately. To evaluate such queries, a ViP2P peer looks up in the network for views which may be used to answer it. For instance, assume the query $q=/ /$ bibliography//book[contains $\left(.,{ }^{\prime}\right.$ Databases $\left.\left.{ }^{\prime}\right)\right] / /$ author is issued at peer $p_{5}$ (step 1, in Figure 1 left). To process $q, p_{5}$ looks up the keys bibliography, book, 'Databases' and author in the DHT, and retrieves a set of view definitions, $v_{1}, v_{2}$ and $v_{3}$ (step 2). Observe that $q$ can be rewritten as $v_{1} / /$ author; therefore, $p_{5}$ can answer $q$ just by retrieving and extracting $q$ 's results out of $v_{1}$. Alternatively, assume that $q$ can also be rewritten by joining views $v_{2}$ and $v_{3}$ as $\mathrm{ViP} 2 \mathrm{P}$ can combine several views to rewrite a query [4. In that case, $p_{5}$ can retrieve the views $v_{2}$ and $v_{3}$ (step 3) and join them to evaluate $q$. However, the whole content of both views has to be shipped to $p_{5}$ to evaluate the query $q$. Instead, $v_{2}$ can be shipped to peer $p_{1}$, joined locally with $v_{3}$ at $p_{1}$ (step 4), who will send the query results to the query peer (step 4'), avoiding extraneous data transfers.

\section{$3 \quad$ ViP2P Peer Architecture}

Figure 1 (right) outlines the architecture of a ViP2P peer. In this Section, we introduce the auxiliary modules on which every peer relies, and then move to the main modules, which are included in the ViP2P Core box of Figure 1.

Resource Catalog provides the underlying DHT layer used to keep peers connected, and to index and lookup views. It employs the FreePastry DHT, which is an open-source implementation of the Pastry overlay network [10]. It provides efficient request routing, deterministic object location, and load balancing.

Data Exchange module is responsible for all data transfers and relies on Java RMI. Experience with FreePastry has shown that Pastry-routed inter-peer communications quickly become the bottleneck when sending important volumes of data 1. Instead, we use RMI (with our own (de)serialization methods, properly controlling concurrency at the sender and receiver side etc.) to send larger messages containing view tuples, when views are materialized and queried.

Data Storage Within each peer, view tuples are efficiently stored into a native store that we built using the BerkeleyDB2 library. It allows storing, retrieving and sorting entries, with transactional guarantees for concurrent operations.

The VIP2P GUI enables publishing views, documents and evaluating queries. We now describe the core modules.

Document Management determines to which views the peer's documents may contribute data, and extracts and sends this data to the appropriate consumers.

2 http://www.oracle.com/technetwork/database/berkeleydb/ 
- View definition lookup. When a new document is published by a peer, this module looks up in the DHT for view definitions to which the document may contribute data. The result is a superset of view definitions of the views that the document might contribute data to. These definitions are then passed to the view data extraction module.

- View data extraction. Given a list of view definitions, this module at a publisher peer extracts from the document the tuples matching each view, and ships them, in a parallel fashion, to the corresponding consumers. The view data extractor is capable of simultaneously matching several views on a given document, thus extracting the corresponding tuples at a single document traversal.

View Management. This module handles view indexing and materialization.

- View indexing. This module implements the view indexing process. In this context, a given algorithm for extracting (key, value) pairs out of a view definition is termed a view indexing strategy [4. In our experiments, the most efficient is the Label Indexing (LI) strategy, indexing a view $v$ by each $v$ node label (element or attribute name, or word).

- View materialization. The view materialization module receives tuples from remote publishers and stores them in the respective BerkeleyDB database. In a large scale, real-world scenario, thousands of documents might be contributing data to a single view. To avoid overload on its incoming data transfers, this module implements a back-pressure tuple-send/receive protocol which informs the publisher when the consumer is overloaded, so that the publisher waits until the consumer is ready to accept new tuples.

Query Management comprises the following modules for query evaluation.

- View lookup. This module, given a query, performs a lookup in the DHT network retrieving the view definitions that may be used to rewrite the query. Depending on the indexing strategy (mentioned earlier in this Section), this module uses a different view lookup method.

- Query rewriting. Given a query and a set of view definitions, this module produces a logical plan which, evaluated on some views, produces exactly the results required by the query (algorithm detailed in [7]).

- Query optimization. This module receives a logical plan that is output by the query rewriting module, and translates it to an optimized physical plan. The optimization concerns both the logical (join reordering, push selections/projections etc.) and physical (dictating the exact flow of data during query execution) level.

- Query execution. This module provides a set of physical operators which can be executed by any ViP2P peer, implementing the standard iterator-based execution model. Since ViP2P is a distributed application, operators can be deployed to peers and executed in a distributed manner. The query optimization module is the one to decide the parts of a physical plan that every peer executes. 


\section{Experimental Results}

We now present a set of experiments studying ViP2P performance, carried on the Grid5000 infrastructure 3 . Due to space limitations, we only report here on our main findings; many more experiments are described in [4.

In our experiments, we used synthetic "product catalog" documents of controllable size (more details can be found in [4). First, all views are created and indexed. Then, on a signal sent to all publishers, these peers start publishing all their documents as fast as possible. This is a "flash crowd" scenario, aiming at stress-testing our system. Queries are posed and processed after all the views are filled with data. Section 4.1 examines view materialization, while Section 4.2 studies the performance of the query execution engine.

\subsection{View Materialization in Large Networks}

We present three materialization experiments; many more can be found in 44.

Experiment 1: One Publisher, Varying Data Size, 64 Consumers. In this experiment we study how materialization time is affected when the total size of published data is increased. We use one publisher holding all the data in the network. The size of the published data varies from 64MBs to 1024MBs.

Each of the 64 consumers holds one view of the form $/ /$ catalog $/ /$ camera $_{K}$ cont where $K$ varies according to the peer that holds the view. For example, the first consumer holds the view $/ /$ catalog $/ /$ camera $_{1}$ cont, the second holds the view $/ /$ catalog $/ /$ camera $_{2}$ cont etc. This way, from each document the publisher extracts 64 tuples, each of which is sent to a different consumer. All the content of the documents is absorbed by the 64 views.

We run two variations of the same experiment: $(i)$ one for sequential tuple sending where a publisher sends the tuples to their corresponding consumers one after the other, and (ii) one for parallel tuple sending, where a publisher ships the tuples to their corresponding consumers simultaneously. The graph at left in Figure 2 shows, as expected, that the materialization time increases linearly with the size of data published in the network in both cases. It also shows that the materialization time in the case of parallel tuple sending is considerably shorter (about $3000 \mathrm{sec}$. instead of $11500 \mathrm{sec}$. for absorbing 1024MBs of data).

Experiment 2: 64 Publishers, Varying Data Size, One Consumer. We now focus on the impact of the number of (simultaneous) publishers on the capacity of absorbing the data into a single view. The published data size varies from $64 \mathrm{MBs}$ to $3.2 \mathrm{GBs}$, and is equally distributed to 64 publishers. All the published data ends up in one view. Similarly to Experiment 1, we test 2 modes of tuple-receiving concurrency: $(i)$ sequential tuple receiving and; (ii) parallel tuple receiving.

Figure 2 (center) depicts the materialization time as the size of the published data increases. We observe that the materialization time increases proportionally to the size of published data in both sequential and parallel tuple receiving

3 https://www.grid5000.fr 

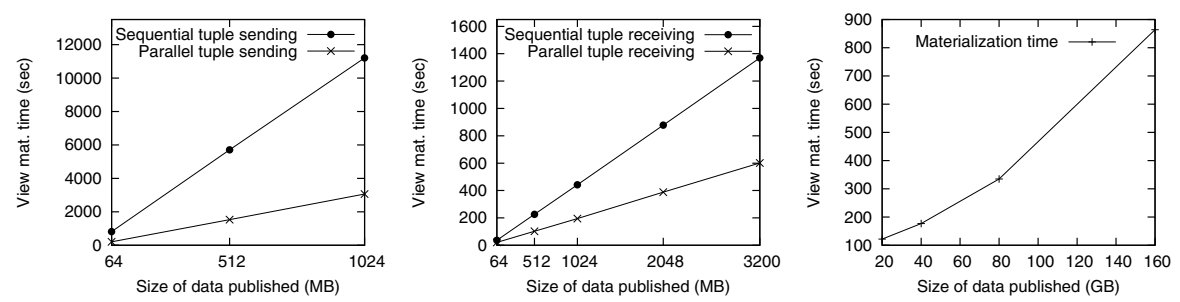

Fig. 2. Experiment 1: one publisher, varying size of data, 64 consumers (left); Experiment 2: 64 publishers, varying data size, one consumer (center); Experiment 3: publishing varying size of data in 50 groups of 5 peers each (right).

modes. Also, parallel tuple receiving reduces the view materialization time by more than $50 \%$ (600 sec. instead of about $1400 \mathrm{sec}$. to absorb 3.2GBs of data).

From the two graphs (left, center) in Figure 2, we conclude that it is faster for the network to absorb data using one consumer and many publishers rather than many consumers and one publisher since it is slow for a peer to extract all the available data by itself and ship them to the consumers.

Experiment 3: Community Publishing. A "community publishing" setting is the closest to real world scenarios: a large and complex environment, with many publishers and many consumers. We use a network of 250 peers, each of which holds the same number of $1 \mathrm{MB}$ documents. We logically divide the network into 50 groups of 5 publishers and one consumer each. The data of all publishers in a group is of interest only to the consumer of that group.The total amount of data published (and shipped to the views) varies from 20GBs to $160 \mathrm{GBs}$.

Figure 2 (right) shows that the materialization time grows linearly with the published data size. This experiment demonstrates the good scalability properties of ViP2P as the data volume increases. Moreover, it shows that ViP2P exploits many parallelization opportunities in such "community publishing" scenarios when extracting, sending, receiving and storing view tuples. Here we report on sharing up to $160 \mathrm{~GB}$ of data over up to 250 peers with a throughput of $238 \mathrm{MB} / \mathrm{s}$ while KadoP [1] scaled up to $1 \mathrm{~GB}$ of data over 50 peers with a throughput of $0.33 \mathrm{MB} / \mathrm{s}$ and psiX [9] used $262 \mathrm{MBs}$ of data and 11 computers.

\subsection{Query Engine Evaluation}

In this Section, we investigate the query processing performance as the data size increases. We use 20 publisher peers, two of which are also consumers, while another publisher is a query peer. The query peer and the two consumers are located in three different French cities. The number of published documents varies from 20 to 500; all documents contribute to the views.

The document used in this experiment is the same as in the previous experiments with a slight difference: its root element catalog has only one child, named camera. The views defined in the network are the following: 

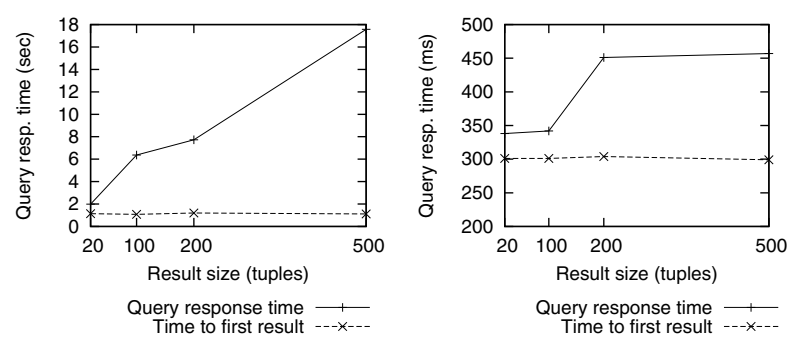

Fig. 3. Query execution time vs. number of result tuples for $q_{1}$ (left) and $q_{2}$ (right)

- $v_{1}$ is $/ /$ catalog $_{I D} / /$ camera $_{I D} / /$ description $_{I D, \text { cont }}$

- $v_{2}$ is $/ /$ catalog $_{I D} / /$ camera $_{I D} / /$ description $_{I D}$, price $_{I D, v a l}$, specs $\left._{I D, \text { cont }}\right\}$

Each document contributes a tuple to each view. The tuples of $v_{1}$ are large in size, since the description element is the largest element in our documents. A $v_{2}$ tuple is quite smaller since it does not store the full camera descriptions. We use two queries: $q_{1}$ asks for the description cont $_{\text {, }}$ specs $_{\text {cont }}$ and price $_{\text {val }}$ of each camera. To evaluate $q_{1}, \mathrm{ViP} 2 \mathrm{P}$ joins the views $v_{1}$ and $v_{2}$. Observe that $q_{1}$ returns full XML elements, and in particular, product descriptions, which are voluminous in our data set. Therefore, $q_{1}$ returns roughly all the published data (from $10 \mathrm{MB}$ in 20 tuples, to $250 \mathrm{MB}$ in 500 tuples). $q_{2}$ requires the description $I D$, specs $_{I D}$ and price $_{I D}$ of each camera. It is very similar to $q_{1}$ with but it can be answered based on $v_{2}$ only. The returned data is much smaller since there are only IDs and no XML elements: from $2 \mathrm{~KB}$ in 20 tuples, to $40 \mathrm{~KB}$ in 500 tuples.

Figure 3 shows the query response time and the time to get the first result for the two queries. The low selectivity query $q_{1}$ in Figure 3 (left) takes longer than $q_{2}$ (right), due to the larger data transfers and the necessary view join. The time to first result is always constant for both $q_{1}$ and $q_{2}$ and does not depend on the result size. For $q_{1}$, a hash join is used to combine $v_{1}$ and $v_{2}$, and thus no tuple is output before the view $v_{2}$ has been built into the buckets of the hash join. This is done around one second in the case of $q_{1}$ and about $300 \mathrm{~ms}$ for $q_{2}$.

The ViP2P query processing engine scales quite linearly answering queries in a wide-area network. The fact that ViP2P rewrites queries into logical plans which are then passed to an optimizer, enables it to take advantage of known optimization techniques used in XML and/or distributed databases.

\section{Conclusion and Perspectives}

We have presented the ViP2P platform for building and maintaining structured materialized views, and processing queries using the views in a DHT network. Our experiments show that ViP2P outperforms similar systems by several orders of magnitude, in particular for the data publication throughput and the overall volume of data published. Many more experiments are described in our technical 
report 4]. We currently investigate a distributed version of our automatic view selection algorithm [5]. We also consider multiple-level subscriptions, where some views could be filled with data based on lower-level views.

Acknowledgements. We experimented on Grid'5000 (https://www.grid 5000.fr). We thank A. Tilea, J. Camacho-Rodríguez, A. Roatis, V. Mishra and J. Leblay for their help. This work was partially supported by ANR 08-DEFIS-004.

\section{References}

1. Abiteboul, S., Manolescu, I., Polyzotis, N., Preda, N., Sun, C.: XML processing in DHT networks. In: ICDE (2008)

2. Bonifati, A., Cuzzocrea, A.: Storing and retrieving XPath fragments in structured P2P networks. Data Knowl. Eng. 59(2) (2006)

3. Galanis, L., Wang, Y., Jeffery, S.R., DeWitt, D.J.: Locating Data Sources in Large Distributed Systems. In: VLDB (2003)

4. Karanasos, K., Katsifodimos, A., Manolescu, I., Zoupanos, S.: The ViP2P Platform: Views in P2P. Research Report $\mathrm{N}^{\circ} 7812$ (November 2011)

5. Katsifodimos, A., Manolescu, I., Vassalos, V.: Materialized View Selection for XQuery Workloads. In: SIGMOD (to appear, 2012)

6. Lillis, K., Pitoura, E.: Cooperative XPath caching. In: SIGMOD (2008)

7. Manolescu, I., Karanasos, K., Vassalos, V., Zoupanos, S.: Efficient XQuery rewriting using multiple views. In: ICDE (2011)

8. Miliaraki, I., Kaoudi, Z., Koubarakis, M.: XML Data Dissemination Using Automata on Top of Structured Overlay Networks. In: WWW (2008)

9. Rao, P.R., Moon, B.: Locating XML documents in a peer-to-peer network using distributed hash tables. IEEE TKDE 21 (2009)

10. Rowstron, A., Druschel, P.: Pastry: Scalable, distributed object location and routing for large-scale peer-to-peer systems. In: ICDSP (November 2001)

11. Skobeltsyn, G., Hauswirth, M., Aberer, K.: Efficient Processing of XPath Queries with Structured Overlay Networks. In: Meersman, R., Tari, Z. (eds.) CoopIS/DOA/ODBASE 2005. LNCS, vol. 3761, pp. 1243-1260. Springer, Heidelberg (2005) 